VOL. 21, No. 2 (2017), 75-84

\title{
General integral formulas involving Humbert hypergeometric functions of two variables
}

\author{
Ahmed Ali Atash and Hussein Saleh Bellehaj
}

Abstract. In this paper, we have established two general integral formulas involving Humbert hypergeometric functions of two variables $\Phi_{2}$ and $\Psi_{2}$. The results are obtained with the help of a generalization of classical Kummer's summation theorem on the sum of the series ${ }_{2} F_{1}(-1)$ due to Lavoie et al. [5]. Some interesting applications are also presented.

\section{INTRODUCTION}

The confluent hypergeometric functions $\Phi_{2}$ and $\Psi_{2}$ are defined and represented as follows $[2,8]$ :

$$
\Phi_{2}\left[a, a^{\prime} ; b ; x ; y\right]=\sum_{m, n=0}^{\infty} \frac{(a)_{m}\left(a^{\prime}\right)_{n} x^{m} y^{n}}{(b)_{m+n} m ! n !}
$$

and

$$
\Psi_{2}\left[a ; b, b^{\prime} ; x ; y\right]=\sum_{m, n=0}^{\infty} \frac{(a)_{m+n} x^{m} y^{n}}{(b)_{m}\left(b^{\prime}\right)_{n} m ! n !},
$$

where $(\lambda)_{n}$ denotes the Pochhammer symbol defined by [8]

$$
(\lambda)_{n}= \begin{cases}1, & \text { if } n=0 \\ \lambda(\lambda+1) \ldots(\lambda+n-1), & \text { if } n=1,2,3, \ldots\end{cases}
$$

Exton $[3,4]$ gave the definitions and the Laplace integral representations of the quadruple hypergeometric functions $K_{5}$ and $K_{12}$ as follows:

2010 Mathematics Subject Classification. 33C05, 33C65, 33C70.

Key words and phrases. Humbert functions, Exton functions, Kampé de Fériet function, Integral formulas, Kummer's theorem. 


$$
\begin{aligned}
K_{5} & \left(a, a, a, a ; b_{1}, b_{1}, b_{2}, b_{2} ; c_{1}, c_{2}, c_{3}, c_{4} ; x, y, z, t\right) \\
\quad & =\sum_{p, q, r, s=0}^{\infty} \frac{(a)_{p+q+r+s}\left(b_{1}\right)_{p+q}\left(b_{2}\right)_{r+s} x^{p} y^{q} z^{r} t^{s}}{\left(c_{1}\right)_{p}\left(c_{2}\right)_{q}\left(c_{3}\right)_{r}\left(c_{4}\right)_{s} p ! q ! r ! s !} \\
& =\frac{1}{\Gamma(a)} \int_{0}^{\infty} e^{-s} s^{a-1} \Psi_{2}\left(b_{1} ; c_{1}, c_{2} ; x s, y s\right) \Psi_{2}\left(b_{2} ; c_{3}, c_{4} ; z s, t s\right) \mathrm{d} s
\end{aligned}
$$

and

$$
\begin{aligned}
& K_{12}\left(a, a, a, a ; b_{1}, b_{2}, b_{3}, b_{4} ; c_{1}, c_{1}, c_{2}, c_{2} ; x, y, z, t\right) \\
& \quad=\sum_{p, q, r, s=0}^{\infty} \frac{(a)_{p+q+r+s}\left(b_{1}\right)_{p}\left(b_{2}\right)_{q}\left(b_{3}\right)_{r}\left(b_{4}\right)_{s} x^{p} y^{q} z^{r} t^{s}}{\left(c_{1}\right)_{p+q}\left(c_{2}\right)_{r+s} p ! q ! r ! s !} \\
& \quad=\frac{1}{\Gamma(a)} \int_{0}^{\infty} e^{-s} s^{a-1} \Phi_{2}\left(b_{1}, b_{2} ; c_{1} ; x s, y s\right) \Phi_{2}\left(b_{3}, b_{4} ; c_{2} ; z s, t s\right) \mathrm{d} s .
\end{aligned}
$$

The Kampé de Fériet function of two variables $F_{l: m ; n}^{p: q ; k}[x, y]$ is defined and represented as follows [8]:

$$
\begin{aligned}
& F_{l: m ; n}^{p: q ; k}\left[\begin{array}{c}
\left(a_{p}\right):\left(b_{q}\right) ;\left(c_{k}\right) ; x, y \\
\left(\alpha_{l}\right):\left(\beta_{m}\right) ;\left(\gamma_{n}\right) ;
\end{array}\right] \\
& =\sum_{r, s=0}^{\infty} \frac{\prod_{j=1}^{p}\left(a_{j}\right)_{r+s} \prod_{j=1}^{q}\left(b_{j}\right)_{r} \prod_{j=1}^{k}\left(c_{j}\right)_{s}}{\prod_{j=1}^{l}\left(\alpha_{j}\right)_{r+s} \prod_{j=1}^{m}\left(\beta_{j}\right)_{r} \prod_{j=1}^{n}\left(\gamma_{j}\right)_{s}} \frac{x^{r}}{r !} \frac{y^{s}}{s !} .
\end{aligned}
$$

In the present investigation, we shall require the following generalization of the classical Kummer's theorem for the series ${ }_{2} F_{1}(-1)$ [5]:

$$
\begin{array}{r}
{ }_{2} F_{1}\left[\begin{array}{cc}
a, b & ; \\
1+a-b+i & ;
\end{array}\right]=\frac{\Gamma\left(\frac{1}{2}\right) \Gamma(1+a-b+i) \Gamma(1-b)}{2^{a} \Gamma\left(1-b+\frac{1}{2}(i+|i|)\right)} \\
\times\left\{\frac{A_{i}}{\Gamma\left(\frac{1}{2} a+\frac{1}{2} i+\frac{1}{2}-\left[\frac{1+i}{2}\right]\right) \Gamma\left(1+\frac{1}{2} a-b+\frac{1}{2} i\right)}\right. \\
\left.+\frac{B_{i}}{\Gamma\left(\frac{1}{2} a+\frac{1}{2} i-\left[\frac{i}{2}\right]\right) \Gamma\left(\frac{1}{2}+\frac{1}{2} a-b+\frac{1}{2} i\right)}\right\}
\end{array}
$$

for $(i=0, \pm 1, \pm 2, \pm 3, \pm 4, \pm 5)$, where $[x]$ denotes the greatest integer less than or equal to $x$ and $|x|$ denotes the usual absolute value of $x$. The coefficients $A_{i}$ and $B_{i}$ are given respectively in [5]. When $i=0,(1.7)$ reduces immediately to the classical Kummer's theorem [1],(see also [6])

$$
{ }_{2} F_{1}\left[\begin{array}{c}
a, b ; \\
1+a-b ;
\end{array}\right]=\frac{\Gamma(1+a-b) \Gamma\left(\frac{1}{2}\right)}{2^{a} \Gamma\left(1+\frac{1}{2} a-b\right) \Gamma\left(\frac{1}{2} a+\frac{1}{2}\right)} .
$$


The following results will be required also [8]:

$$
\begin{gathered}
(\alpha)_{n}=\frac{\Gamma(\alpha+n)}{\Gamma(\alpha)}, \frac{\Gamma(\alpha-n)}{\Gamma(\alpha)}=\frac{(1-)^{n}}{(1-\alpha)_{n}}, \\
\Gamma\left(\frac{1}{2}\right) \Gamma(1+\alpha)=2^{\alpha} \Gamma\left(\frac{1}{2}+\frac{1}{2} \alpha\right) \Gamma\left(1+\frac{1}{2} \alpha\right), \\
(\alpha)_{2 n}=2^{2 n}\left(\frac{1}{2} \alpha\right)_{n}\left(\frac{1}{2} \alpha+\frac{1}{2}\right)_{n}
\end{gathered}
$$

and

$$
(2 n) !=2^{2 n}\left(\frac{1}{2}\right)_{n} n !,(2 n+1) !=2^{2 n}\left(\frac{3}{2}\right)_{n} n ! .
$$

\section{Main Integrals Formulas}

\section{First Integral}

$$
\begin{aligned}
\frac{1}{\Gamma(a)} \int_{0}^{\infty} e^{-s} s^{a-1} \Psi_{2}\left(b_{1} ; c_{1}, c_{1}+i ; x s,-x s\right) \Psi_{2}\left(b_{2} ; c_{2}, c_{2}+i ; y s,-y s\right) \mathrm{d} s \\
=\sum_{m_{1}=0}^{\infty} \sum_{m_{2}=0}^{\infty} \frac{(a)_{2 m_{1}+2 m_{2}}\left(b_{1}\right)_{2 m_{1}}\left(b_{2}\right)_{2 m_{2}} x^{2 m_{1}} y^{2 m_{2}}}{\left(c_{1}\right)_{2 m_{1}}\left(c_{2}\right)_{2 m_{2}}\left(2 m_{1}\right) !\left(2 m_{2}\right) !} \\
\quad \times\left(A_{i}^{(1)} C_{1}+B_{i}^{(1)} D_{1}\right)\left(A_{i}^{(2)} C_{2}+B_{i}^{(2)} D_{2}\right) \\
+\sum_{m_{1}=0}^{\infty} \sum_{m_{2}=0}^{\infty} \frac{(a)_{2 m_{1}+2 m_{2}+1}\left(b_{1}\right)_{2 m_{1}+1}\left(b_{2}\right)_{2 m_{2}} x^{2 m_{1}+1} y^{2 m_{2}}}{\left(c_{1}\right)_{2 m_{1}+1}\left(c_{2}\right)_{2 m_{2}}\left(2 m_{1}+1\right) !\left(2 m_{2}\right) !} \\
\quad \times\left(A_{i}^{(3)} E_{1}+B_{i}^{(3)} F_{1}\right)\left(A_{i}^{(2)} C_{2}+B_{i}^{(2)} D_{2}\right) \\
+\sum_{m_{1}=0}^{\infty} \sum_{m_{2}=0}^{\infty} \frac{(a)_{2 m_{1}+2 m_{2}+1}\left(b_{1}\right)_{2 m_{1}}\left(b_{2}\right)_{2 m_{2}+1} x^{2 m_{1}} y^{2 m_{2}+1}}{\left(c_{1}\right)_{2 m_{1}}\left(c_{2}\right)_{2 m_{2}+1}\left(2 m_{1}\right) !\left(2 m_{2}+1\right) !} \\
\times\left(A_{i}^{(1)} C_{1}+B_{i}^{(1)} D_{1}\right)\left(A_{i}^{(4)} E_{2}+B_{i}^{(4)} F_{2}\right) \\
+\sum_{m_{1}=0}^{\infty} \sum_{m_{2}=0}^{\infty} \frac{(a)_{2 m_{1}+2 m_{2}+2}\left(b_{1}\right)_{2 m_{1}+1}\left(b_{2}\right)_{2 m_{2}+1} x^{2 m_{1}+1} y^{2 m_{2}+1}}{\left(c_{1}\right)_{2 m_{1}+1}\left(c_{2}\right)_{2 m_{2}+1}\left(2 m_{1}+1\right) !\left(2 m_{2}+1\right) !} \\
\times\left(A_{i}^{(3)} E_{1}+B_{i}^{(3)} F_{1}\right)\left(A_{i}^{(4)} E_{2}+B_{i}^{(4)} F_{2}\right),
\end{aligned}
$$

where

$$
C_{r}=\frac{2^{2 m_{r}} \Gamma\left(\frac{1}{2}\right) \Gamma\left(c_{r}+i\right) \Gamma\left(c_{r}+2 m_{r}\right)}{\Gamma\left(c_{r}+2 m_{r}+\frac{1}{2}(i+|i|)\right) \Gamma\left(-m_{r}+\frac{1}{2} i+\frac{1}{2}-\left[\frac{1+i}{2}\right]\right) \Gamma\left(m_{r}+c_{r}+\frac{1}{2} i\right)}
$$




$$
\begin{gathered}
D_{r}=\frac{2^{2 m_{r}} \Gamma\left(\frac{1}{2}\right) \Gamma\left(c_{r}+i\right) \Gamma\left(c_{r}+2 m_{r}\right)}{\Gamma\left(c_{r}+2 m_{r}+\frac{1}{2}(i+|i|)\right) \Gamma\left(-m_{r}+\frac{1}{2} i-\left[\frac{i}{2}\right]\right) \Gamma\left(m_{r}+c_{r}-\frac{1}{2}+\frac{1}{2} i\right)} \\
E_{r}=\frac{2^{2 m_{r}+1} \Gamma\left(\frac{1}{2}\right) \Gamma\left(c_{r}+i\right) \Gamma\left(c_{r}+2 m_{r}+1\right)}{\Gamma\left(c_{r}+2 m_{r}+1+\frac{1}{2}(i+|i|)\right) \Gamma\left(-m_{r}+\frac{1}{2} i-\left[\frac{1+i}{2}\right]\right) \Gamma\left(m_{r}+\frac{1}{2}+c_{r}+\frac{1}{2} i\right)} \\
F_{r}=\frac{2^{2 m_{r}+1} \Gamma\left(\frac{1}{2}\right) \Gamma\left(c_{r}+i\right) \Gamma\left(c_{r}+2 m_{r}+1\right)}{\Gamma\left(c_{r}+2 m_{r}+1+\frac{1}{2}(i+|i|)\right) \Gamma\left(-m_{r}-\frac{1}{2}+\frac{1}{2} i-\left[\frac{i}{2}\right]\right) \Gamma\left(m_{r}+c_{r}+\frac{1}{2} i\right)}
\end{gathered}
$$

(for $i=0, \pm 1, \pm 2, \pm 3, \pm 4, \pm 5 ; r=1,2$ ).

The coefficients $A_{i}^{(1)}$ and $B_{i}^{(1)}$ can be obtained from the tables of $A_{i}$ and $B_{i}$ given in [5] by replacing $a$ and $b$ by $-2 m_{1}$ and $1-c_{1}-2 m_{1}$, the coefficients $A_{i}^{(2)}$ and $B_{i}^{(2)}$ can be obtained from the tables of $A_{i}$ and $B_{i}$ by replacing $a$ and $b$ by $-2 m_{2}$ and $1-c_{2}-2 m_{2}$, the coefficients $A_{i}^{(3)}$ and $B_{i}^{(3)}$ can be obtained from the tables of $A_{i}$ and $B_{i}$ by replacing $a$ and $b$ by $-2 m_{1}-1$ and $-c_{1}-2 m_{1}$ and the coefficients $A_{i}^{(4)}$ and $B_{i}^{(4)}$ can be obtained from the tables of $A_{i}$ and $B_{i}$ by replacing $a$ and $b$ by $-2 m_{2}-1$ and $-c_{2}-2 m_{2}$.

\section{Second Integral}

$$
\begin{aligned}
& \frac{1}{\Gamma(a)} \int_{0}^{\infty} e^{-s} s^{a-1} \Phi_{2}\left(b_{1}-i, b_{1} ; c_{1} ; x s,-x s\right) \Phi_{2}\left(b_{2}-i, b 2 ; c_{2} ; y s,-y s\right) \mathrm{d} s \\
& =\sum_{m_{1}=0}^{\infty} \sum_{m_{2}=0}^{\infty} \frac{(a)_{2 m_{1}+2 m_{2}}\left(b_{1}-i\right)_{2 m_{1}}\left(b_{2}-i\right)_{2 m_{2}} x^{2 m_{1}} y^{2 m_{2}}}{\left(c_{1}\right)_{2 m_{1}}\left(c_{2}\right)_{2 m_{2}}\left(2 m_{1}\right) !\left(2 m_{2}\right) !} \\
& \quad \times\left(A_{i}^{\prime(1)} C_{1}^{\prime}+B_{i}^{\prime(1)} D_{1}^{\prime}\right)\left(A_{i}^{\prime(2)} C_{2}^{\prime}+B_{i}^{\prime(2)} D_{2}^{\prime}\right) \\
& +\sum_{m_{1}=0}^{\infty} \sum_{m_{2}=0}^{\infty} \frac{(a)_{2 m_{1}+2 m_{2}+1}\left(b_{1}-i\right)_{2 m_{1}+1}\left(b_{2}-i\right)_{2 m_{2}} x^{2 m_{1}+1} y^{2 m_{2}}}{\left(c_{1}\right)_{2 m_{1}+1}\left(c_{2}\right)_{2 m_{2}}\left(2 m_{1}+1\right) !\left(2 m_{2}\right) !} \\
& +\sum_{m_{1}=0}^{\infty} \sum_{m_{2}=0}^{\infty} \frac{(a)_{2 m_{1}+2 m_{2}+1}\left(b_{1}-i\right)_{2 m_{1}}\left(b_{2}-i\right)_{2 m_{2}+1} x^{2 m_{1}} y^{2 m_{2}+1}}{\left(c_{1}\right)_{2 m_{1}}\left(c_{2}\right)_{2 m_{2}+1}\left(2 m_{1}\right) !\left(2 m_{2}+1\right) !} \\
& \times\left(A_{i}^{\prime(1)} C_{1}^{\prime}+B_{i}^{\prime(1)} D_{1}^{\prime}\right)\left(A_{i}^{\prime(4)} E_{2}^{\prime}+B_{i}^{\prime(4)} F_{2}^{\prime}\right) \\
& +\sum_{m_{1}=0}^{\infty} \sum_{m_{2}=0}^{\infty} \frac{(a)_{2 m_{1}+2 m_{2}+2}\left(b_{1}-i\right)_{2 m_{1}+1}\left(b_{2}-i\right)_{2 m_{2}+1} x^{2 m_{1}+1} y^{2 m_{2}+1}}{\left(c_{1}\right)_{2 m_{1}+1}\left(c_{2}\right)_{2 m_{2}+1}\left(2 m_{1}+1\right) !\left(2 m_{2}+1\right) !} \\
& \times\left(A_{i}^{\prime(3)} E_{1}^{\prime}+B_{i}^{\prime(3)} F_{1}^{\prime}\right)\left(A_{i}^{\prime(4)} E_{2}^{\prime}+B_{i}^{\prime(4)} F_{2}^{\prime}\right),
\end{aligned}
$$


where

$$
\begin{aligned}
C_{r}^{\prime} & =\frac{2^{2 m_{r}} \Gamma\left(\frac{1}{2}\right) \Gamma\left(1-2 m_{r}-b_{r}+i\right) \Gamma\left(1-b_{r}\right)}{\Gamma\left(1-b_{r}+\frac{1}{2}(i+|i|)\right) \Gamma\left(-m_{r}+\frac{1}{2} i+\frac{1}{2}-\left[\frac{1+i}{2}\right]\right) \Gamma\left(1-m_{r}-b_{r}+\frac{1}{2} i\right)} \\
D_{r}^{\prime} & =\frac{2^{2 m_{r}} \Gamma\left(\frac{1}{2}\right) \Gamma\left(1-2 m_{r}-b_{r}+i\right) \Gamma\left(1-b_{r}\right)}{\Gamma\left(1-b_{r}+\frac{1}{2}(i+|i|)\right) \Gamma\left(-m_{r}+\frac{1}{2} i-\left[\frac{i}{2}\right]\right) \Gamma\left(-m_{r}+\frac{1}{2}-b_{r}+\frac{1}{2} i\right)} \\
E_{r}^{\prime} & =\frac{2^{2 m_{r}+1} \Gamma\left(\frac{1}{2}\right) \Gamma\left(-2 m_{r}-b_{r}+i\right) \Gamma\left(1-b_{r}\right)}{\Gamma\left(1-b_{r}+\frac{1}{2}(i+|i|)\right) \Gamma\left(-m_{r}+\frac{1}{2} i-\left[\frac{1+i}{2}\right]\right) \Gamma\left(-m_{r}+\frac{1}{2}-b_{r}+\frac{1}{2} i\right)} \\
F_{r}^{\prime} & =\frac{2^{2 m_{r}+1} \Gamma\left(\frac{1}{2}\right) \Gamma\left(-2 m_{r}-b_{r}+i\right) \Gamma\left(1-b_{r}\right)}{\Gamma\left(1-b_{r}+\frac{1}{2}(i+|i|)\right) \Gamma\left(-m_{r}-\frac{1}{2}+\frac{1}{2} i-\left[\frac{i}{2}\right]\right) \Gamma\left(-m_{r}-b_{r}+\frac{1}{2} i\right)}
\end{aligned}
$$

for $i=0, \pm 1, \pm 2, \pm 3, \pm 4, \pm 5 ; r=1,2$.

The coefficients $A_{i}^{\prime(1)}$ and $B_{i}^{\prime(1)}$ can be obtained from the tables of $A_{i}$ and $B_{i}$ given in [5] by replacing $a$ by $-2 m_{1}$, the coefficients $A_{i}^{\prime(2)}$ and $B_{i}^{\prime(2)}$ can be obtained from the tables of $A_{i}$ and $B_{i}$ by replacing $a$ by $-2 m_{2}$, the coefficients $A_{i}^{\prime(3)}$ and $B_{i}^{\prime(3)}$ can be obtained from the tables of $A_{i}$ and $B_{i}$ by replacing $a$ by $-2 m_{1}-1$ and the coefficients $A_{i}^{\prime(4)}$ and $B_{i}^{\prime(4)}$ can be obtained from the tables of $A_{i}$ and $B_{i}$ by replacing $a$ by $-2 m_{2}-1$.

\section{Proof of the first integral:}

Denoting the left hand side of (2.1) by $I$, then from the definition (1.4), we have

$$
I=\sum_{m_{1}, p_{1}, m_{2}, p_{2}=0}^{\infty} \frac{(a)_{m_{1}+p_{1}+m_{2}+p_{2}}\left(b_{1}\right)_{m_{1}+p_{1}}\left(b_{2}\right)_{m_{2}+p_{2}} x^{m_{1}}(-x)^{p_{1}} y^{m_{2}}(-y)^{p_{2}}}{\left(c_{1}\right)_{m_{1}}\left(c_{1}+i\right)_{p_{1}}\left(c_{2}\right)_{m_{2}}\left(c_{2}+i\right)_{p_{2}} m_{1} ! p_{1} ! m_{2} ! p_{2} !}
$$

Now, using the well-known results [8]

$$
\begin{gathered}
(\alpha)_{m+n}=(\alpha)_{m}(\alpha+m)_{n} \\
\sum_{m=0}^{\infty} \sum_{n=0}^{\infty} A(n, m)=\sum_{m=0}^{\infty} \sum_{n=0}^{m} A(n, m-n), \\
(\alpha)_{m-n}=\frac{(-1)^{n}(\alpha)_{m}}{(1-\alpha-m)_{n}}, 0 \leq n \leq m \\
(m-n) !=\frac{(-1)^{n} m !}{(-m)_{n}}, 0 \leq n \leq m,
\end{gathered}
$$

then after a little simplification, we have

$$
I=\sum_{m_{1}=0}^{\infty} \sum_{m_{2}=0}^{\infty} \frac{(a)_{m_{1}+m_{2}}\left(b_{1}\right)_{m_{1}}\left(b_{2}\right)_{m_{2}} x^{m_{1}} y^{m_{2}}}{\left(c_{1}\right)_{m_{1}}\left(c_{2}\right)_{m_{2}} m_{1} ! m_{2} !}
$$




$$
{ }_{2} F_{1}\left[\begin{array}{cc}
-m_{1}, 1-c_{1}-m_{1} & ; \\
c_{1}+i & ;
\end{array}\right]{ }_{2} F_{1}\left[\begin{array}{cc}
-m_{2}, 1-c_{2}-m_{2} & ; \\
c_{2}+i & ;
\end{array}\right]
$$

Separating into its even and odd terms, we have

$$
\begin{aligned}
& I=\sum_{m_{1}=0}^{\infty} \sum_{m_{2}=0}^{\infty} \frac{(a)_{2 m_{1}+2 m_{2}}\left(b_{1}\right)_{2 m_{1}}\left(b_{2}\right)_{2 m_{2}} x^{2 m_{1}} y^{2 m_{2}}}{\left(c_{1}\right)_{2 m_{1}}\left(c_{2}\right)_{2 m_{2}}\left(2 m_{1}\right) !\left(2 m_{2}\right) !} \\
& \times{ }_{2} F_{1}\left[\begin{array}{cc}
-2 m_{1}, 1-c_{1}-2 m_{1} ; \\
c_{1}+i & ;
\end{array}\right]{ }_{2} F_{1}\left[\begin{array}{cc}
-2 m_{2}, 1-c_{2}-2 m_{2} ; \\
c_{2}+i & ;
\end{array}\right] \\
& +\sum_{m_{1}=0 m_{2}=0}^{\infty} \sum_{(a)_{2 m_{1}+2 m_{2}+1}\left(b_{1}\right)_{2 m_{1}+1}\left(b_{2}\right)_{2 m_{2}} x^{2 m_{1}+1} y^{2 m_{2}}}^{\left(c_{1}\right)_{2 m_{1}+1}\left(c_{2}\right)_{2 m_{2}}\left(2 m_{1}+1\right) !\left(2 m_{2}\right) !} \\
& \times{ }_{2} F_{1}\left[\begin{array}{cc}
-2 m_{1}-1,-c_{1}-2 m_{1} ; \\
c_{1}+i & ;
\end{array}\right]{ }_{2} F_{1}\left[\begin{array}{cc}
-2 m_{2}, 1-c_{2}-2 m_{2} ; \\
c_{2}+i & ;
\end{array}\right] \\
& +\sum_{m_{1}=0 m_{2}=0}^{\infty} \sum_{(a)_{2 m_{1}+2 m_{2}+1}\left(b_{1}\right)_{2 m_{1}}\left(b_{2}\right)_{2 m_{2}+1} x^{2 m_{1}} y^{2 m_{2}+1}}^{\left(c_{1}\right)_{2 m_{1}}\left(c_{2}\right)_{2 m_{2}+1}\left(2 m_{1}\right) !\left(2 m_{2}+1\right) !} \\
& \times_{2} F_{1}\left[\begin{array}{cc}
-2 m_{1}, 1-c_{1}-2 m_{1} ; \\
c_{1}+i & ;
\end{array}\right]{ }_{2} F_{1}\left[\begin{array}{cc}
-2 m_{2}-1,-c_{2}-2 m_{2} ; \\
c_{2}+i & ;
\end{array}\right] \\
& +\sum_{m_{1}=0}^{\infty} \sum_{m_{2}=0}^{\infty} \frac{(a)_{2 m_{1}+2 m_{2}+2}\left(b_{1}\right)_{2 m_{1}+1}\left(b_{2}\right)_{2 m_{2}+1} x^{2 m_{1}+1} y^{2 m_{2}+1}}{\left(c_{1}\right)_{2 m_{1}+1}\left(c_{2}\right)_{2 m_{2}+1}\left(2 m_{1}+1\right) !\left(2 m_{2}+1\right) !} \\
& \times_{2} F_{1}\left[\begin{array}{cc}
-2 m_{1}-1,-c_{1}-2 m_{1} ; \\
c_{1}+i & ;
\end{array}\right]{ }_{2} F_{1}\left[\begin{array}{cc}
-2 m_{2}-1,-c_{2}-2 m_{2} ; \\
c_{2}+i & ;
\end{array}\right] \text {. }
\end{aligned}
$$

Finally, if we use the generalized Kummer's theorem (1.7), then, after a little simplification, we readily arrive at the right hand side of (2.1).This completes the proof of the first integral . The proof of the second integral is similar to that of the first integral with the only difference that we use here the result (1.5). 


\section{Applications}

(i) In (2.1), if we take $i=0$ and use the results (1.9)-(1.12), we get after a little simplification the following integral formula:

$$
\begin{aligned}
& \frac{1}{\Gamma(a)} \int_{0}^{\infty} e^{-s} s^{a-1} \Psi_{2}\left(b_{1} ; c_{1}, c_{1} ; x s,-x s\right) \Psi_{2}\left(b_{2} ; c_{2}, c_{2} ; y s,-y s\right) \mathrm{d} s \\
& =F_{0: 3 ; 3}^{2: 2 ; 2}\left[\begin{array}{c}
\frac{1}{2} a, \frac{1}{2} a+\frac{1}{2}: \frac{1}{2} b_{1}, \frac{1}{2} b_{1}+\frac{1}{2} ; \frac{1}{2} b_{2}, \frac{1}{2} b_{2}+\frac{1}{2} ;-4 x^{2},-4 y^{2} \\
-\quad: c_{1}, \frac{1}{2} c_{1}, \frac{1}{2} c_{1}+\frac{1}{2} ; c_{2}, \frac{1}{2} c_{2}, \frac{1}{2} c_{2}+\frac{1}{2} ;
\end{array}\right] .
\end{aligned}
$$

Now, in (3.1) using the result [7]

$$
\Psi_{2}(a ; b, b ; x,-x)={ }_{2} F_{3}\left[\begin{array}{c}
\frac{1}{2} a, \frac{1}{2} a+\frac{1}{2} ; \\
b, \frac{1}{2} b, \frac{1}{2} b+\frac{1}{2} ;
\end{array}-x^{2}\right],
$$

we get

$$
\begin{gathered}
\frac{1}{\Gamma(a)} \int_{0}^{\infty} e^{-s} s^{a-1}{ }_{2} F_{3}\left[\begin{array}{c}
\frac{1}{2} b_{1}, \frac{1}{2} b_{1}+\frac{1}{2} ;-(x s)^{2} \\
c_{1}, \frac{1}{2} c_{1}, \frac{1}{2} c_{1}+\frac{1}{2} ;
\end{array}\right] \\
\left.\times{ }_{2} F_{3}\left[\begin{array}{c}
\frac{1}{2} b_{2}, \frac{1}{2} b_{2}+\frac{1}{2} ; \\
c_{2}, \frac{1}{2} c_{2}, \frac{1}{2} c_{2}+\frac{1}{2} ;
\end{array}\right](y s)^{2}\right] \mathrm{d} s \\
=F_{0: 3 ; 3}^{2: 2 ; 2}\left[\begin{array}{c}
\frac{1}{2} a, \frac{1}{2} a+\frac{1}{2}: \frac{1}{2} b_{1}, \frac{1}{2} b_{1}+\frac{1}{2} ; \frac{1}{2} b_{2}, \frac{1}{2} b_{2}+\frac{1}{2} ;-4 x^{2},-4 y^{2} \\
-\quad: c_{1}, \frac{1}{2} c_{1}, \frac{1}{2} c_{1}+\frac{1}{2} ; c_{2}, \frac{1}{2} c_{2}, \frac{1}{2} c_{2}+\frac{1}{2} ;
\end{array}\right],
\end{gathered}
$$

which for $c_{1}=b_{1}, c_{2}=b_{2}$ reduces to the following integral in terms of Appell function $F_{4}[8]$

$$
\begin{array}{r}
\frac{1}{\Gamma(a)} \int_{0}^{\infty} e^{-s} s^{a-1}{ }_{0} F_{1}\left(-; b_{1} ;-x^{2} s^{2}\right)_{0} F_{1}\left(-; b_{2} ;-y^{2} s^{2}\right) \mathrm{d} s \\
=F_{4}\left[\frac{1}{2} a, \frac{1}{2} a+\frac{1}{2} ; b_{1}, b_{2} ;-4 x^{2},-4 y^{2}\right] .
\end{array}
$$

(ii) In (2.1), if we take $i=1$ and use the results (1.9)-(1.12), we get after a little simplification the following integral formula:

$$
\begin{aligned}
& \frac{1}{\Gamma(a)} \int_{0}^{\infty} e^{-s} s^{a-1} \Psi_{2}\left(b_{1} ; c_{1}, c_{1}+1 ; x s,-x s\right) \Psi_{2}\left(b_{2} ; c_{2}, c_{2}+1 ; y s,-y s\right) \mathrm{d} s
\end{aligned}
$$

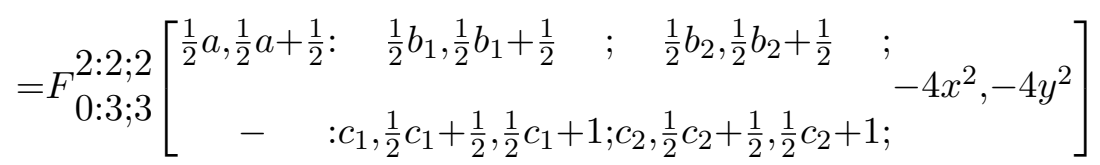

$$
\begin{aligned}
& +\frac{a b_{1} x}{c_{1}\left(c_{1}+1\right)}
\end{aligned}
$$




$$
\begin{aligned}
& \times F_{0: 3 ; 3}^{2: 2 ; 2}\left[\begin{array}{c}
\frac{1}{2} a+\frac{1}{2}, \frac{1}{2} a+1: \quad \frac{1}{2} b_{1}+\frac{1}{2}, \frac{1}{2} b_{1}+1 \quad ; \quad \frac{1}{2} b_{2}, \frac{1}{2} b_{2}+\frac{1}{2} \quad ; \\
-\quad: c_{1}+1, \frac{1}{2} c_{1}+1, \frac{1}{2} c_{1}+\frac{3}{2} ; c_{2}, \frac{1}{2} c_{2}+\frac{1}{2}, \frac{1}{2} c_{2}+1 ;
\end{array}\right] \\
& +\frac{a b_{2} y}{c_{2}\left(c_{2}+1\right)} \\
& \times F_{0: 3 ; 3}^{2: 2 ; 2}\left[\begin{array}{c}
\frac{1}{2} a+\frac{1}{2}, \frac{1}{2} a+1: \quad \frac{1}{2} b_{1}, \frac{1}{2} b_{1}+\frac{1}{2} \quad ; \quad \frac{1}{2} b_{2}+\frac{1}{2}, \frac{1}{2} b_{2}+1 ; \\
-\quad: c_{1}, \frac{1}{2} c_{1}+\frac{1}{2}, \frac{1}{2} c_{1}+1 ; c_{2}+1, \frac{1}{2} c_{2}+1, \frac{1}{2} c_{2}+\frac{3}{2} ;
\end{array}\right] \\
& +\frac{a(a+1) b_{1} b_{2} x y}{c_{1} c_{2}\left(c_{1}+1\right)\left(c_{2}+1\right)}
\end{aligned}
$$

$\left.\times F_{0: 3 ; 3}^{2: 2 ; 2}\left[\begin{array}{c}\frac{1}{2} a+1, \frac{1}{2} a+\frac{3}{2}: \quad \frac{1}{2} b_{1}+\frac{1}{2}, \frac{1}{2} b_{1}+1 \quad ; \quad \frac{1}{2} b_{2}+\frac{1}{2}, \frac{1}{2} b_{2}+1 \quad ; \\ -\quad: c_{1}+1, \frac{1}{2} c_{1}+1, \frac{1}{2} c_{1}+\frac{3}{2} ; c_{2}+1, \frac{1}{2} c_{2}+1, \frac{1}{2} c_{2}+\frac{3}{2} ;\end{array}\right] .4 x^{2},-4 y^{2}\right]$.

Further, taking $c_{1}=b_{1}-1, c_{2}=b_{2}-1$ in (3.5), we get

$$
\begin{aligned}
\frac{1}{\Gamma(a)} \int_{0}^{\infty} e^{-s} s^{a-1} \Psi_{2}\left(b_{1} ; b_{1}-1, b_{1} ; x s,-x s\right) \Psi_{2}\left(b_{2} ; b_{2}-1, b_{2} ; y s,-y s\right) \mathrm{d} s \\
=F_{4}\left\lfloor\frac{1}{2} a, \frac{1}{2} a+\frac{1}{2} ; b_{1}-1, b_{2}-1 ;-4 x^{2},-4 y^{2}\right\rfloor \\
+\frac{a x}{b_{1}-1} F_{4}\left[\frac{1}{2} a+\frac{1}{2}, \frac{1}{2} a+1 ; b_{1}, b_{2}-1 ;-4 x^{2},-4 y^{2}\right] \\
+\frac{a y}{b_{2}-1} F_{4}\left[\frac{1}{2} a+\frac{1}{2}, \frac{1}{2} a+1 ; b_{1}-1, b_{2} ;-4 x^{2},-4 y^{2}\right] \\
+\frac{a(a+1) x y}{\left(b_{1}-1\right)\left(b_{2}-1\right)} F_{4}\left[\frac{1}{2} a+1, \frac{1}{2} a+\frac{3}{2} ; b_{1}, b_{2} ;-4 x^{2},-4 y^{2}\right] .
\end{aligned}
$$

(iii) In (2.2), if we take $i=0$ and use the results (1.9)-(1.12), we get after a little simplification the following integral formula:

$$
\begin{aligned}
& \frac{1}{\Gamma(a)} \int_{0}^{\infty} e^{-s} s^{a-1} \Phi_{2}\left(b_{1}, b_{1} ; c_{1} ; x s,-x s\right) \Phi_{2}\left(b_{2}, b_{2} ; c_{2} ; y s,-y s\right) \mathrm{d} s \\
& =F_{0: 2 ; 2}^{2: 1 ; 1}\left[\begin{array}{c}
\frac{1}{2} a, \frac{1}{2} a+\frac{1}{2}: \quad b_{1} \quad ; \quad b_{2} \quad ; \\
-\quad: \frac{1}{2} c_{1}, \frac{1}{2} c_{1}+\frac{1}{2} ; \frac{1}{2} c_{2}, \frac{1}{2} c_{2}+\frac{1}{2} ;
\end{array}\right] .
\end{aligned}
$$


Further, taking $c_{1}=2 b_{1}, c_{2}=2 b_{2}$ in (3.7) and using the result [7]

$$
\Phi_{2}(a, a ; 2 a ; x, y)=e^{y}{ }_{1} F_{1}(a ; 2 a ; x,-y),
$$

we get

$$
\begin{gathered}
\frac{1}{\Gamma(a)} \int_{0}^{\infty} e^{-s-x s-y s} s^{a-1}{ }_{1} F_{1}\left(b_{1} ; 2 b_{1} ; 2 x s\right)_{1} F_{1}\left(b_{2} ; 2 b_{2} ; 2 y s\right) \mathrm{d} s \\
=F_{4}\left[\frac{1}{2} a, \frac{1}{2} a+\frac{1}{2} ; b_{1}+\frac{1}{2}, b_{2}+\frac{1}{2} ; x^{2}, y^{2}\right] .
\end{gathered}
$$

(iv) In (2.2), if we take $i=1$ and use the results (1.9)-(1.12), we get after a little simplification the following integral formula:

$$
\begin{aligned}
& \frac{1}{\Gamma(a)} \int_{0}^{\infty} e^{-s} s^{a-1} \Phi_{2}\left(b_{1}-1, b_{1} ; c_{1} ; x s,-x s\right) \Phi_{2}\left(b_{2}-1, b_{2} ; c_{2} ; y s,-y s\right) \mathrm{d} s \\
& =F_{0: 2 ; 2}^{2: 1 ; 1}\left[\begin{array}{c}
\frac{1}{2} a, \frac{1}{2} a+\frac{1}{2}: \quad b_{1} \quad ; \quad b_{2} \quad ; \quad x^{2}, y^{2} \\
-\quad: \frac{1}{2} c_{1}, \frac{1}{2} c_{1}+\frac{1}{2} ; \frac{1}{2} c_{2}, \frac{1}{2} c_{2}+\frac{1}{2} ;
\end{array}\right] \\
& -\frac{a x}{c_{1}} F_{0: 2 ; 2}^{2: 1 ; 1}\left[\begin{array}{ccc}
\frac{1}{2} a+\frac{1}{2}, \frac{1}{2} a+1: & b_{1} \quad ; \quad b_{2} \quad ; \\
- & : \frac{1}{2} c_{1}+\frac{1}{2}, \frac{1}{2} c_{1}+1 ; \frac{1}{2} c_{2}, \frac{1}{2} c_{2}+\frac{1}{2} ;
\end{array}\right] \\
& -\frac{a y}{c_{2}} F_{0: 2 ; 2}^{2: 1 ; 1}\left[\begin{array}{cccc}
\frac{1}{2} a+\frac{1}{2}, \frac{1}{2} a+1: & b_{1} \quad ; \quad b_{2} \quad ; \\
- & : \frac{1}{2} c_{1}, \frac{1}{2} c_{1}+\frac{1}{2} ; \frac{1}{2} c_{2}+\frac{1}{2}, \frac{1}{2} c_{2}+1 ;
\end{array}\right] \\
& +\frac{a(a+1) x y}{c_{1} c_{2}}
\end{aligned}
$$

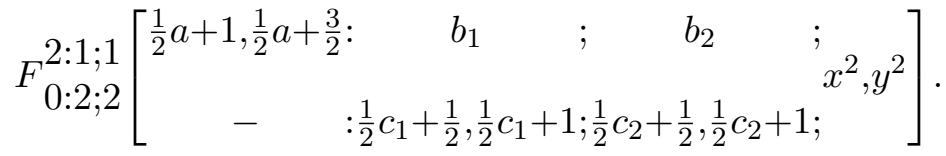

Further, taking $c_{1}=2 b_{1}-1, c_{2}=2 b_{2}-1$ in $(3.10)$, we get

$$
\begin{aligned}
\frac{1}{\Gamma(a)} \int_{0}^{\infty} e^{-s} s^{a-1} \Phi_{2}\left(b_{1}-1, b_{1} ; 2 b_{1}-1 ; x s,-x s\right) \\
\times \Phi_{2}\left(b_{2}-1, b_{2} ; 2 b_{2}-1 ; y s,-y s\right) \mathrm{d} s \\
=F_{4}\left\lfloor\frac{1}{2} a, \frac{1}{2} a+\frac{1}{2} ; b_{1}-\frac{1}{2}, b_{2}-\frac{1}{2} ; x^{2}, y^{2}\right\rfloor \\
-\frac{a x}{2 b_{1}-1} F_{4}\left[\frac{1}{2} a+\frac{1}{2}, \frac{1}{2} a+1 ; b_{1}+\frac{1}{2}, b_{2}-\frac{1}{2} ; x^{2}, y^{2}\right]
\end{aligned}
$$




$$
\begin{aligned}
& -\frac{a y}{2 b_{2}-1} F_{4}\left[\frac{1}{2} a+\frac{1}{2}, \frac{1}{2} a+1 ; b_{1}-\frac{1}{2}, b_{2}+\frac{1}{2} ; x^{2}, y^{2}\right] \\
& +\frac{a(a+1) x y}{\left(2 b_{1}-1\right)\left(2 b_{2}-1\right)} F_{4}\left[\frac{1}{2} a+1, \frac{1}{2} a+\frac{3}{2} ; b_{1}+\frac{1}{2}, b_{2}+\frac{1}{2} ; x^{2}, y^{2}\right] .
\end{aligned}
$$

The other special cases of the integrals (2.1) and (2.2) can also be obtained in the similar manner.

\title{
REFERENCES
}

[1] W. N. Bailey, Generalized Hypergeometric Series, Cambridge University Press, Cambridge, 1935 .

[2] A. Erdélyi, W. Magnus, F. Oberhettinger and F. G. Tricomi , Higher Transcendental Functions, Vol. I, McGraw-Hill, New York, Toronto and London, 1953.

[3] H. Exton, Certain hypergeometric functions of four variables, Bull. Soc. Math. Gréce,(N.S.) 13 (1972), $104-113$.

[4] H. Exton, Multiple Hypergeometric Functions and Applications, John Wiley and Sons (Halsted Press), New York, 1976.

[5] J. L. Lavoie, F. Grondin and A. K. Rathie, Generalizations of Whipple's theorem on the sum of $a_{3} F_{2}$, Journal of Computational and Applied Mathematics, 72 (1996), $293-300$.

[6] E. D. Rainville, Special Functions, The Macmillan Company, New York, 1960.

[7] H. M. Srivastava and P. W. Karlsson, Multiple Gaussian Hypergeometric series, Halsted Press , New York, 1985.

[8] H. M. Srivastava and H. L. Manocha, A Treatise on Generating Functions, Halsted Press, New York, 1984.

\author{
Ahmed Ali Atash \\ Department of Mathematics \\ FACUlTy of EduCATiOn-ShabWah \\ Aden University \\ ADEN \\ YEMEN \\ E-mail address: ah-a-atash@hotmail.com \\ Hussein Saleh Bellehaj \\ Department of Mathematics \\ FACUlty of EducAtion-Shabwa \\ Aden University \\ ADEN \\ YEMEN
}

